\title{
COVID-19 in diabetic patients; impacts and implications
}

\author{
Mohaddeseh Bahmani $^{1^{\mathbb{D}}}$, Rojin Chegini ${ }^{1}$, Hamid Nasri ${ }^{1,2^{*}}{ }^{(\mathbb{C}}$ \\ ${ }^{1}$ Nickan Research Institute, Isfahan, Iran \\ ${ }^{2}$ Department of Nephrology, Isfahan University of Medical Sciences, Isfahan, Iran
}

Correspondence to:

Prof. Hamid Nasri, hamidnasri@

yahoo.com,

hamidnasri@med.mui.ac.ir

Received: 7 Oct. 2020

Accepted: 16 Dec. 2020

ePublished: 20 Dec. 2020

Citation: Bahmani M,

Chegini R, Nasri $\mathrm{H}$. COVID-19 in diabetic patients; impacts and implications. J Prev Epidemiol. 2020;5(2):e29. doi: $10.34172 /$ jpe.2020.29

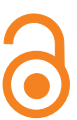

\begin{abstract}
The main aim of this study is to review the general characteristics of this virus and its relationship with diabetes according to studies that have been done about this relevance. We investigated and searched related articles to this topic in EBSCO, Medline/PubMed, Scopus, Web of Science, Embase, Directory of Open Access Journals (DOAJ) and Google Scholar. According to numerous studies, diabetes is currently recognized as a risk factor for further complications of COVID-19.

Keywords: COVID-19, SARS-CoV-2, Diabetes, Obesity
\end{abstract}

\section{Introduction}

Diabetes mellitus is a chronic disorder with a significant impact on many people, families, and communities (1). It is divided into four categories. Type one is the result of the misfunctioning of insulin-generating beta cells, type two is caused by insulin resistance, the third type is gestational diabetes mellitus which is diagnosed in the second or third trimester of pregnancy, and the fourth type occurs for other reasons, including disorders of the exocrine pancreas. Type 2 is the most prevalent, representing $90-95 \%$ of cases. However, patients with diabetes of any type are at risk for chronic complications (2). There were 463 million adult patients with diabetes worldwide according to the International Diabetes Federation (IDF), in 2019. This number is anticipated to reach 700 million by 2045 (3). It is one of the foremost reasons for disability with a global impact (1). The new coronavirus, called 2019-nCoV (2019 novel coronavirus) or SARS-CoV-2 (severe acute respiratory syndrome coronavirus 2), which initially emerged in Wuhan city, China, at the end of 2019, is the responsible cause of COVID-19 (coronavirus disease 2019) (4). Its spread was declared as a pandemic on March 11, 2020, by the World Health Organization (WHO) (5). Thereafter, cases were found within 201 countries by the end of March 2020 (6). Till August 6, 2020, more than 18 million infected individuals have been reported (4). At present, it has a global impact and represents a threat to people around the

\section{Key point}

Recent studies showed that diabetes is currently a risk factor for further complications of COVID-19. Therefore, diabetic patients should ensure to receive the necessary and routine care they need.

world and global economics with a humanto-human contagion (4). According to the studies, cardiovascular and cerebrovascular comorbidities, high blood pressure, diabetes, advanced age, (7) cancer, chronic kidney or respiratory diseases, would expose the patient to a higher risk for developing complications of the novel coronavirus infection (8). Several studies have reported the association between diabetes and increased COVID-19 severity (9-13). Lifestyle changes after emergence of the novel virus and lockdowns to control its rate of spread have resulted in reduced physical activity, including in diabetic patients, which may prevent these individuals from receiving the routine care they require (14). Regarding these reports and since diabetes is prevalent in most parts of the globe (8), in this study we review the general characteristics of this virus and its relationship with diabetes taking into consideration studies have been done in this regard so far.

\section{Materials and Methods}

Articles related to this topic were searched in EBSCO, Medline/PubMed, Scopus, Web of Science, Embase, Directory of Open Access Journals (DOAJ) and Google Scholar, using 
the following keywords or their combinations; COVID-19, severe acute respiratory syndrome coronavirus 2 (SARS-CoV-2), COVID-19 severity, COVID-19 risk factors, diabetes, obesity, pregnancy, cancer, chronic renal failure and angiotensin-converting enzyme 2

\section{COVID-19 characteristics}

The new coronavirus is a beta-coronavirus belonging to the Coronaviridae family, the large single-stranded RNA viruses. Beta coronaviruses can be pathogenic to mammals including humans (15). Previous virulent beta coronaviruses that emerged throughout the last two decades, were MERS-CoV (Middle East respiratory syndrome coronavirus) and SARS-CoV (severe acute respiratory syndrome coronavirus) (15). The most common symptoms are fatigue, cough, shortness of breath, and fever. However, taste and olfactory disorders, headache, and hemoptysis are additionally reported (15). In addition to pulmonary involvement, renal, cardiovascular, and liver involvement may take place (8).

Pulmonary involvement may lead to acute respiratory disease, resulting in subsequently respiratory and multiorgan failure and finally death (16). Younger patients fairly have mild symptoms (15). An incubation period of between 2 and 14 days has been reported by the USA CDC. The disease has a zoonotic origin; however, it is currently transmitted by direct contact with aerosols or droplets from an infected individual to others or touching contaminated surfaces and objects (8). The person may be asymptomatic and the likelihood of becoming symptomatic and eventually the need for hospitalization increases with age (16). Although this infection is seen among all ages, the mean age is about 50 years (8). The general fatality rate of SARS-CoV-2 is low (1.4-2.3\%) (17). While some conditions are associated with higher mortality rates. According to the reports by China CDC from evaluating the first 72314 infected individuals, men were more likely to die than women. For people who were already healthy, the fatality rate was $0.9 \%, 6.3 \%$ in patients with a past medical history of chronic respiratory diseases, $10.5 \%$ in cardiovascular patients, $7.3 \%$ in diabetics, $5.6 \%$ for cancer, and $6.0 \%$ for hypertension. The mortality rate of severely ill patients was $49.0 \%$ (18). Generally, according to the US CDC, patients with chronic renal failure, sickle cell anemia, a body mass index (BMI) of $>30 \mathrm{~kg} / \mathrm{m}^{2}$, organ transplantation, diabetes mellitus, cardiovascular co-morbidities, cancer, chronic obstructive pulmonary disease, smokers, and pregnant women are at a higher risk in the current pandemic (19).

\section{COVID-19 pathogenesis}

Similar SARS-CoV, the door to the 2019-nCoV entry to the cell is ACE2 (angiotensin-converting enzyme 2) (15), an integral glycoprotein of type one in the membrane (17). Via the receptor-binding domain in the S1 subunit of viral Spike protein, the virus binds to this receptor. Merging to the cellular membrane arises via the S2 segment. Proximal serine proteases, like TMPRSS2, are involved in spike cleavage and S protein priming. Finally, viral RNA enters the cytosol and replication develops the constitution of the mature virion and then spreads. This receptor is present on the epithelial cells of the intestine, blood vessels, kidneys, and lungs (20). What contributes to developing the severe forms of the disease is the "cytokine storm", which is associated with events such as impaired iron homeostasis leading to hyperferritinemia, oxidative stress, a chief key in the disease intensity and pathogenesis, and finally coagulopathy (21). According to the results of a study on 138 hospitalized patients, factors associated with higher mortality included acute renal impairment, cytokine storm syndrome, and over-activation of the coagulation system (7). According to one other study, patients with more severe pulmonary involvement had higher levels of inflammatory mediators such as interleukin-6 (IL-6) and more severe lymphopenia (22). IL-6 is expressed for a longer time than other inflammatory mediators such as TNF-a (tumor necrosis factor) and is a prognostic factor in all diseases that cause the cytokine storm (23). CRP (C-reactive protein), IL-6, and more elevated d-dimer levels are related to the disease severity (8). Exaggerated production of these pro-inflammatory cytokines, TNF- $\alpha$, IL-6, and IL-1, befalls in SARS-CoV-2 patients with acute respiratory distress syndrome (ARDS) (24). Infiltration of the inflammatory cell in the alveolar cells is another responsible mechanism. Hemorrhagic necrosis and disseminated microthrombi are seen in lung biopsy specimens of the individuals after death (25). The adaptive immune reaction against the virus is not however established (26).

The kinin-kallikrein system has an impression on the pathophysiology of ARDS. The final products of this system are kinins (like kallidin and bradykinin). This system can have a role in viral-induced pulmonary damage as well as its other manifestations and may also be one of the therapeutic approaches (27).

\section{Disorders in diabetic patients}

Diabetes is associated with micro and macro-vascular complications (8). Type two diabetes is a leading cause of premature death, cardiovascular complications, and chronic renal failure (28). Hypersensitivity of diabetic patients to other infections such as Staphylococcus aureus has been observed, and also has been proven as a risk factor for increased SARS-CoV mortality. Type 2diabetes is a chronic, low-level inflammatory disorder accompanied by immune imbalances, metabolic syndrome, and obesity. Obesity is associated with an increased systemic and local release of chemokines and inflammatory agents. Besides, the number and activation of adaptive and innate immune cells in the adipose tissue are heightened (23). In diabetics, T-helper 1 related response is delayed and they have higher levels of inflammatory cytokines such as TNF- $\alpha$, IL-6, 8, 
and 1 (29).

Diabetic patients are reported to have a poor prognosis involving other viral infections; like pandemic influenza A H1N1 and MERS. One of the reasons could be the innate immune system deficiency, which results from hyperglycemia (24). Any infection may induce morbidity in diabetic patients (30). Non-controlled glycemic status affects the immune system. The response of cytokines to pathogens is more severe in diabetics. T-helper 17 cells have a higher percentage, while other $\mathrm{T}$ cells are diminished. In addition, infection detracts from the proper production of insulin and its effects (31). High blood glucose affects the function of macrophages as well as granulocytes. Increased mortality in people with type 1 or 2 diabetes for any causes has been seen since the onset of this novel virus. The association betwixt HbA1c levels and SARS-CoV2 fatality has been independently observed (32). Diabetes increases the tendency to clot formation. COVID-19induced hypoxia inures dysfunction of the endothelium and eventually raises the risk of thromboembolic events. As a result of developing lymphopenia in the course of this disease, the effect of T cells in inhibiting the cytokine formation will be detracted. Cytokine storm which is the result of these inflammatory agents' over-production is more vigorous in diabetic patients (33).

Diabetes and the new pandemic

It is not yet clear if diabetes or its complications add susceptibility to COVID-19 or would be an independent risk factor for poor outcomes (28).

In one study in the city of Wuhan at the beginning of this infection in January 2020 on 904 patients with clinical features or a positive nucleic acid test of SARS-CoV-2, the most common co-morbidity was hypertension followed by diabetes. In this study, higher levels of CRP and advanced age were independent risk factors for higher mortality rates, although hypertension was not associated with worse outcomes. In diabetic patients, the rate of secondary infection, inflammatory response, and acute renal damage were higher. Comparing those treated with insulin and those not on this medication, there was no significant difference in developing severe forms of the disease, but the first group had higher inflammatory markers (CRP and procalcitonin) and fewer albumin levels. In contrast, patients taking metformin had lower interleukin-6 and blood urea nitrogen and higher albumin levels (34). In the study by Guo et al, in diabetic patients, pneumonia was more severe than non-diabetics according to their computed tomography imaging scores. The severity of organ damage was higher in diabetics and they had more elevated markers of organ damage such as $\mathrm{LDH}$ and GGT. Additionally, lower levels of total proteins, including albumin, indicated malnutrition. Lymphopenia and neutrophilia were more drastic. Affecting the glucose metabolism, the virus increased the patients need for insulin (23).
In a Chinese meta-analysis, diabetes or high blood pressure was twice more prevalent in severe cases than non-severe cases, but there wasn't a heightened susceptibility to getting infected; hence the prevalence of these co-morbidities amongst infected individuals did not differ from the general population (35).

One other study showed the impact of glucose homeostasis on the disease outcomes. Patients with chronic renal failure had worse outcomes may be due to their underlying vascular complications (30). In another study of 450 SARS-CoV-2 patients, diabetics had 1.6 folds need for hospitalization in the ICU and a twice early mortality rate (36). Diabetes is also linked to several known other 2019-nCoV severity risk factors, including cardiovascular diseases, hypertension, being elderly, and male gender (37). According to the recent study in the UK on 61414470 people and a total number of 23698 SARS-CoV2 pertaining mortalities, any types of diabetes (including one-third of all in-hospital SARS-CoV-2 mortalities) was accompanied by a heightened mortality rate during hospitalization, after adjusting for other related variables such as ethnicity or gender and even cardiovascular diseases; but not for patients past drug history. Type one was related to a vaster risk than other types. Likewise, other infections are more common in people with type one diabetes than type two (38).

Up-regulation of ACE2 occurs in the chronic use of ACE inhibitors or ARBs, which are used in the management of proteinuria, hypertension, and diabetes (28) in additionally with thiazolidinediones. Moreover, hypertension, cerebral stroke, and diabetes mellitus may be related to ACE2 polymorphisms chiefly in Asian people. This may increase the person's susceptibility to the virus (20). Statins and glucagon-like peptide-1 agonists such as liraglutide may also upregulate this receptor (39). In an Italian case series, diabetic patients who were treated with insulin at the time of admission had more complications. This may be due to the effect of this drug on the immune function. Patients on DPP4-I had a better outcome, possibly following its role in prohibiting the "cytokine storm" by preventing the DPP4/ CD26 inflammatory pathway (30). Although patients on metformin use have shown better outcomes, it should not be used due to the risk of lactic acidosis in patients with severe renal, cardiovascular, or pulmonary dysfunction (33). In another cohort study in China on 7,337 patients, $13 \%$ of whom had a history of type 2 diabetes. Diabetic patients experienced higher rates of DIC (disseminated intravascular coagulation), heart damage, septic shock, ARDS, and acute renal damage. They showed higher levels of lactate dehydrogenase, IL-6, and CRP. The mortality rate of these individuals was higher. Patients who had better glycemic control had a better prognosis (a suggested target blood glucose of 3.9-10.0 mmol/L). Although there was no adjustment for other comorbidities. One of the reasons mentioned for these events would be immune dysregulation. In people with diabetes, there are disorders 
in the immune system; for example, neutrophilia, and lymphopenia seen in SARS-CoV-2 infection are more drastic in these people. There is also a disturbance in diffusion capacity and volumes of the lungs and additionally in noradrenergic nerves of the pulmonary system (9). Studies have shown that the expression of ACE2 is lower in diabetic patients. This should deduct the risk of these patients become infected with SARS-CoV-2. In general, the exact role of this receptor in increasing the severity of the disease is still unknown. Besides, joining the ACE2 in metabolic organs, the virus contributes to impaired glucose metabolism, leading to several complications like ketoacidosis (29). Moreover, according to studies, as beta cells in the pancreas present the ACE- 2 receptor, the effects of the virus and inflammatory cytokines on them may cause post-infection-onset diabetes (40). As a whole, immune disorders, patients' past drug history, and underlying diseases might be involving factors for developing worse outcomes in diabetic individuals.

\section{Conclusion}

According to many studies, diabetes is currently recognized as a risk factor for further complications of COVID-19. Because the disease is rapidly transmitted and is a pandemic, it is essential to follow the principles of prevention outlined by the World Health Organization to prevent contracting the virus, especially in people with diabetes. Therefore, diabetics should ensure to receive the necessary and routine care they need.

Authors' contribution

Primary draft by RC and MD. HN conducted the edit. All authors read and signed the final manuscript.

Conflicts of interest

The author declared no potential conflicts of interest with respect to the research, authorship, and/or publication of this article.

Ethical considerations

Ethical issues (including plagiarism, data fabrication, double publication) have been completely observed by the authors.

\section{Funding/Support}

None.

\section{References}

1. Diabetes. [cited 2020 Nov 28]. Available from: https://www. who.int/news-room/fact-sheets/detail/diabetes

2. Association AD. Classification and diagnosis of diabetes: Standards of Medical Care in Diabetes-2020. Diabetes Care. 2020;43:S14-31.

3. Saeedi P, Petersohn I, Salpea P, Malanda B, Karuranga S, Unwin $\mathrm{N}$, et al. Global and regional diabetes prevalence estimates for 2019 and projections for 2030 and 2045: Results from the International Diabetes Federation Diabetes Atlas, 9th edition. Diabetes Res Clin Pract. 2019;157:107843.

4. Wor;d Health Organization. WHO Coronavirus Disease (COVID-19) Dashboard. [cited 2020 Nov 28]. https://covid19. who.int/?gclid=CjwKCAjwnK36BRBVEiwAsMT8WJ3y00_
BUzvrLsvbl3uthuoTH_Occ45gyEUbpYRyEqAzIl3aZB6TYxoCcM0QAvD_BwE.

5. Mahase E. Covid-19: WHO declares pandemic because of "alarming levels" of spread, severity, and inaction. BMJ. 2020;368:m1036 doi:10.1136/bmj.m1036.

6. World Health Organisation. Coronavirus disease (COVID-19) situation reports in Malaysia. World Health Organisation; 2020 [cited 2020 Nov 28]. Available from: https://www.who. int/emergencies/diseases/novel-coronavirus-2019/situationreports\%0Ahttps://www.who.int/malaysia/emergencies/ coronavirus-disease-(covid-19)-in-malaysia/covid-19situation-reports-in-malaysia.

7. Wang D, Hu B, Hu C, Zhu F, Liu X, Zhang J, et al. Clinical Characteristics of 138 Hospitalized Patients With 2019 Novel Coronavirus-Infected Pneumonia in Wuhan, China. JAMA. 2020;323(11):1061-9. doi: 10.1001/jama.2020.1585.

8. Hussain A, Bhowmik B, do Vale Moreira NC. COVID-19 and diabetes: Knowledge in progress. Diabetes Res Clin Pract. 2020;162:108142. doi: 10.1016/j.diabres.2020.108142

9. Zhu L, She ZG, Cheng X, Qin JJ, Zhang XJ, Cai J, et al. Association of Blood Glucose Control and Outcomes in Patients with COVID-19 and Pre-existing Type 2 Diabetes. Cell Metab. 2020;31:1068-77.e3. doi: 10.1016/j.cmet.2020.04.021.

10. Bello-Chavolla OY, Bahena-López JP, Antonio-Villa NE, Vargas-Vázquez A, González-Díaz A, Márquez-Salinas A, et al. Predicting Mortality Due to SARS-CoV-2: A Mechanistic Score Relating Obesity and Diabetes to COVID-19 Outcomes in Mexico. J Clin Endocrinol Metab. 2020;105: dgaa346. doi: 10.1210/clinem/dgaa346.

11. Riddle MC, Buse JB, Franks PW, Knowler WC, Ratner RE, Selvin E, et al. COVID-19 in People with Diabetes: Urgently Needed Lessons from Early Reports. Diabetes Care. 2020;43(7):137881. doi: $10.2337 /$ dci20-0024

12. Shi Q, Zhang X, Jiang F, Zhang X, Hu N, Bimu C, et al. Clinical Characteristics and Risk Factors for Mortality of COVID-19 Patients With Diabetes in Wuhan, China: A Two-Center, Retrospective Study. Diabetes Care. 2020;43:1382-91. doi: 10.2337/dc20-0598.

13. Petrilli $\mathrm{CM}$, Jones SA, Yang J, Rajagopalan $\mathrm{H}$, O'Donnell L, Chernyak Y, et al. Factors associated with hospital admission and critical illness among 5279 people with coronavirus disease 2019 in New York City: Prospective cohort study. BMJ. 2020;369. doi: 10.1136/bmj.m1966

14. Abdi A, Jalilian M, Sarbarzeh PA, Vlaisavljevic Z. Diabetes and COVID-19: A systematic review on the current evidences. Diabetes Res Clin Pract. 2020;166:108347. doi: 10.1016/j. diabres.2020.108347.

15. Hu B, Guo H, Zhou P, Shi ZL. Characteristics of SARS-CoV-2 and COVID-19. Nat Rev Microbiol. 2020 Oct 6;1-14. doi: 10.1038/s41579-020-00459-7.

16. Muniyappa R, Gubbi S. COVID-19 pandemic, coronaviruses, and diabetes mellitus. Am J Physiol - Endocrinol Metab. 2020;318:E736-41. doi: 10.1152/ajpendo.00124.2020.

17. Ceriello A. Management of diabetes today: An exciting confusion. Diabetes Res Clin Pract. 2020;162:108129. doi: 10.1016/j.diabres.2020.108129.

18. Surveillances $\mathrm{V}$. The epidemiological characteristics of an outbreak of 2019 novel coronavirus diseases (COVID-19) in China. Zhonghua Liu Xing Bing Xue Za Zhi. 2020;41:145-51.

19. Certain Medical Conditions and Risk for Severe COVID-19 Illness. Centers for Disease Control and Prevention. [cited 2020 Nov 28]. Available from: https://www.cdc.gov/ coronavirus/2019-ncov/need-extra-precautions/people-withmedical-conditions.html.

20. Fang L, Karakiulakis G, Roth M. Are patients with hypertension 
and diabetes mellitus at increased risk for COVID-19 infection? Lancet Respir Med. 2020;8:e21. doi: 10.1016/ S2213-2600(20)30116-8

21. Saleh J, Peyssonnaux C, Singh KK, Edeas M. Mitochondria and microbiota dysfunction in COVID-19 pathogenesis. Mitochondrion. 2020;54:1-7. doi: 10.1016/j.mito.2020.06.008

22. Huang C, Wang Y, Li X, Ren L, Zhao J, Hu Y, et al. Clinical features of patients infected with 2019 novel coronavirus in Wuhan, China. Lancet. 2020;395:497-506. doi: 10.1016/ S0140-6736(20)30183-5.

23. Guo W, Li M, Dong Y, Zhou H, Zhang Z, Tian C, et al. Diabetes is a risk factor for the progression and prognosis of COVID-19. Diabetes Metab Res Rev. 2020;36:e3319. doi: 10.1002/ dmrr.3319. doi: 10.1002/dmrr.3319.

24. Pal R, Bhansali A. COVID-19, diabetes mellitus and ACE2: The conundrum. Diabetes Res Clin Pract. 2020;162:108132. doi: 10.1016/j.diabres.2020.108132

25. Fogarty $\mathrm{H}$, Townsend L, Ni Cheallaigh C, Bergin C, MartinLoeches I, Browne $\mathrm{P}$, et al. COVID19 coagulopathy in Caucasian patients. Br J Haematol. 2020;189:1044-9. doi: 10.1111/bjh.16749

26. Milionis C, Milioni SO. A brief analysis and hypotheses about the risk of COVID-19 for people with type 1 and type 2 diabetes mellitus. J Diabetes Metab Disord. 2020;1-5. doi: 10.1007/s40200-020-00592-3

27. Mohammadi K, Tabatabaee A, Akbari M. Kinin-kallikrein system; a possible pathway responsible for COVID-19. Immunopathol Persa. 2020;7:e11-e11. doi:10.34172/ ipp. 2021.11

28. Selvin E, Juraschek SP. Diabetes epidemiology in the covid-19 pandemic. Diabetes Care. 2020;43:1690-4. doi: 10.2337/ dc20-1295

29. Azar WS, Njeim R, Fares AH, Azar NS, Azar ST, El Sayed M, et al. COVID-19 and diabetes mellitus: how one pandemic worsens the other. Rev Endocr Metab Disord. 2020;21:451-63. doi: 10.1007/s11154-020-09573-6

30. Mirani M, Favacchio G, Carrone F, Betella N, Biamonte E, Morenghi E, et al. Impact of Comorbidities and Glycemia at Admission and Dipeptidyl Peptidase 4 Inhibitors in Patients With Type 2 Diabetes With COVID-19: A Case Series From an Academic Hospital in Lombardy, Italy. Diabetes Care. 2020;43:3042-9.

31. Singh SK, Jain R, Singh S. Vitamin D deficiency in patients with diabetes and COVID- 19 infection. Diabetes Metab Syndr Clin Res Rev. 2020;14:1033-5. doi: 10.1016/j.dsx.2020.06.071

32. Holman N, Knighton P, Kar P, O'Keefe J, Curley M, Weaver A, et al. Risk factors for COVID-19-related mortality in people with type 1 and type 2 diabetes in England: a population-based cohort study. Lancet Diabetes Endocrinol. 2020;8:823-33. doi: 10.1016/S2213-8587(20)30271-0

33. Apicella M, Campopiano MC, Mantuano M, Mazoni L, Coppelli A, Del Prato S. COVID-19 in people with diabetes: understanding the reasons for worse outcomes. Lancet Diabetes Endocrinol. 2020;8:782-92. doi: 10.1016/S22138587(20)30238-2.

34. Chen Y, Yang D, Yang C, Zheng L, Huang K. Clinical characteristics and outcomes of patients with diabetes and covid-19 in association with glucose-lowering medication. Diabetes Care. 2020;43:1399-407. doi: 10.2337/dc20-0660

35. Li B, Yang J, Zhao F, Zhi L, Wang X, Liu L, et al. Prevalence and impact of cardiovascular metabolic diseases on COVID-19 in China. Clin Res Cardiol. 2020;109:531-8. doi: 10.1007/ s00392-020-01626-9

36. Seiglie J, Platt J, Cromer SJ, Bunda B, Foulkes AS, Bassett I V., et al. Diabetes as a risk factor for poor early outcomes in patients hospitalized with COVID-19. Diabetes Care. 2020;43:293844. doi: 10.2337/dc20-1506.

37. Hartmann-Boyce J, Morris E, Goyder C, Kinton J, Perring J, Nunan D, et al. Diabetes and COVID-19: risks, management, and learnings from other national disasters. Diabetes Care. 2020;43):1695-703. doi: 10.2337/dc20-1192

38. Barron E, Bakhai C, Kar P, Weaver A, Bradley D, Ismail H, et al. Associations of type 1 and type 2 diabetes with COVID-19related mortality in England: a whole-population study. Lancet Diabetes Endocrinol. 2020;8(10):813-22. doi: 10.1016/S22138587(20)30272-2

39. Muniyappa R, Gubbi S. COVID-19 pandemic, coronaviruses, and diabetes mellitus. Vol. 318, American Journal of Physiology - Endocrinology and Metabolism. American Physiological Society; 2020. p. E736-41. doi: 10.1152/ajpendo.00124.2020.

40. Gupta R, Misra A. Clinical considerations in patients with diabetes during times of COVID19: An update on lifestyle factors and antihyperglycemic drugs with focus on India. Diabetes Metab Syndr Clin Res Rev. 2020;14:1777-81. doi: 10.1016/j.dsx.2020.09.009 